\title{
'On-farm' seed priming with zinc in chickpea and wheat in Pakistan
}

\section{David Harris • Abdul Rashid • Ghazal Miraj • Muhammad Arif • Muhammed Yunas}

Published online: 15 December 2007

(C) Springer Science + Business Media B.V. 2007

\section{Erratum to: Plant Soil \\ DOI 10.1007/s11104-007-9465-4}

The fourth author's name was incorrect it should have been Muhammad Arif.

The online version of the original article can be found at doi: 10.1007/s11104-007-9465-4.

D. Harris $(\bowtie)$

CAZS Natural Resources, Bangor University,

Gwynedd LL57 2UW, UK

e-mail: d.harris@bangor.ac.uk
A. Rashid · M. Arif · M. Yunas Faculty of Crop Production Sciences,
North West Frontier Province Agricultural University,
Peshawar, Pakistan
G. Miraj
Faculty of Nutrition Sciences,
North West Frontier Province Agricultural University,
Peshawar, Pakistan 